\title{
Corela
}

Cognition, représentation, langage

HS-30 | 2020

Phonétique, littérature et enseignement du FLE : théories et recherches

\section{Magie des sons et de la prosodie, et apprentissage de la phonétique par la poésie : « Le Pont Mirabeau »}

Monique Monville-Burston, Fryni Kakoyianni-Doa et Spyros Armostis

\section{(2) OpenEdition}

Édition électronique

URL : https://journals.openedition.org/corela/10429

DOI : $10.4000 /$ corela.10429

ISSN : $1638-573 \mathrm{X}$

\section{Éditeur}

Université de Poitiers

Référence électronique

Monique Monville-Burston, Fryni Kakoyianni-Doa et Spyros Armostis, « Magie des sons et de la prosodie, et apprentissage de la phonétique par la poésie : «Le Pont Mirabeau »», Corela [En ligne], HS-30 | 2020, mis en ligne le 11 juillet 2020, consulté le 28 juin 2022. URL : http:// journals.openedition.org/corela/10429; DOI : https://doi.org/10.4000/corela.10429

Ce document a été généré automatiquement le 29 septembre 2020.

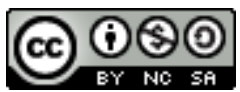

Corela - cognition, représentation, langage est mis à disposition selon les termes de la licence Creative Commons Attribution - Pas d'Utilisation Commerciale - Partage dans les Mêmes Conditions 4.0 International. 


\title{
Démarches et corrections pour une appropriation des textes littéraires dans leur matérialité phonique et écrite par les apprenants de FLE dès le niveau A1 du CECRL
}

\author{
Julie Veldeman-Abry et Dominique Abry
}

Depuis les approches communicatives et la perspective actionnelle, la littérature a retrouvé une certaine légitimité dans l'enseignement du français langue étrangère et seconde comme un des supports indispensables dans une séquence pédagogique.

On constate cependant, d'une part, qu'elle est réservée aux niveaux avancés $B$ et $C$ du Cadre européen commun de référence pour les langues (CECRL) et, d'autre part, qu'elle est utilisée pour travailler à travers la compréhension écrite, essentiellement le lexique, la morphosyntaxe et la culture ( $c f$. Amélie Nothomb, Stupeurs et tremblements, dossier 5, p. 94, Alter ego + B2, Hachette, 2015, ou Jean Giono, « Le voyageur immobile ", La rondeur des jours, unité 10, p. 113, Version originale 4, B2, Maison des langues, 2012). Les textes ne sont jamais enregistrés et dans les propositions d'activités il y a très peu de travail de phonétique véritablement sur les sons et la prosodie, même si le texte est un extrait d'une pièce de théâtre. Par exemple, à partir du texte de Roland Dubillard, «Le plongeon ", Les Nouveaux Diablogues (unité 1, p. 26-27, Saison 4, B2, Didier, 2015), il est simplement proposé de lire, à deux, la scène à voix haute en accentuant les pronoms toniques. Pour l'extrait d'Eugène Ionesco, Le Rhinocéros (dossier 4, p. 76, Alter ego + B1, Hachette, 2013), il est demandé directement de « Jouez cet extrait... ». De même pour le poème d'Émile Verhaeren, «La ville » (dossier 4, p. 76, Alter ego + B2, Hachette, 2015), la consigne est étonnamment succincte : «Lisez le poème à haute voix et donnez-lui le ton qui permettra d'en faire comprendre le sens.»

Une langue, c'est " un cliquetis de sons bizarres », selon Julien Green dans Le langage et son double, qu'il faut s'approprier par une écoute indispensable et une découverte du 
sens qui permettra de transmettre «le plaisir du texte » comme disait Roland Barthes. "Si [l'homme] lit vraiment, s'il y met son savoir en maîtrisant son plaisir, si sa lecture est un acte de sympathie pour l'auditoire comme pour le texte et son auteur, s'il parvient à faire entendre la nécessité d'écrire en réveillant nos plus obscurs besoins de comprendre, alors les livres s'ouvrent grand et la foule de ceux qui se croyaient exclus de la lecture s'engouffre derrière lui. » (Pennac, 1992 : 174)

La lecture à haute voix permet de mesurer l'importance du phonétisme dans un texte. Gustave Flaubert était obsédé par le rythme et la musicalité de chaque phrase. Chaque phrase qu'il avait écrite était soumise à l'épreuve du "gueuloir »: «Une phrase est viable, affirmait-il, quand elle correspond à toutes les nécessités de la respiration. Je sais qu'elle est bonne lorsqu'elle peut être lue tout haut... ». Maupassant, son ami, écrit : "À chaque instant il se levait de sa table, prenait sa feuille de papier l'élevait à la hauteur du regard, et, s'appuyant sur son coude, déclamait, d'une voix mordante et haute. Il écoutait le rythme de sa prose, s'arrêtait pour saisir une sonorité fuyante, combinait les tons, éloignait les assonances, disposait les virgules avec conscience, comme les baltes d'un long chemin.» L'écrivain Akira Mizubayashi, dans Une langue venue d'ailleurs, nous dévoile sa stratégie d'apprentissage du français qu'il parle comme un natif alors qu'il n'a commencé qu'à l'âge de 18 ans : il écoutait des textes littéraires enregistrés par des natifs et nous dit: «Je ne me lassais pas de répéter sans cesse le texte pour le bonheur de l'entendre dans sa matérialité phonique et de lui donner une forme sensible... une majestueuse architecture sonore. »

5 Nous voudrions montrer avec quelques exemples que la littérature est tout à fait adaptée dès le niveau A. Elle est primordiale pour sensibiliser, dans un premier temps, les apprenants à la musicalité de la langue à travers la lecture et surtout l'écoute (il y a de plus en plus de livres enregistrés : $c f$. audiolib.fr) et, dans un second temps, pour les aider à acquérir les sons et la prosodie par un travail de correction phonétique guidé par l'enseignant.

6 Comment amener les apprenants à s'approprier oralement le texte littéraire, le mettre en voix et l'interpréter? Il est nécessaire de faire un travail préparatoire pour les guider et ne pas les mettre en échec. Le texte littéraire est un support riche de sonorités et de musicalité, fruit d'un travail sur le choix des mots et du rythme de la phrase.

7 Nous commencerons avec deux exemples de démarche travaillant la prosodie, plus particulièrement l'intonation linguistique.

\section{Exemple 1}

8 Objectifs :

- rythme-intonation et mise en voix du poème de Mohamed Rouabhi.

- phonie-graphie (lettres muettes).

Niveau : A1.

\section{$1^{\text {re }}$ étape : Discrimination et répétition}

10 Écoutez les deux phrases (question et déclaration) pour bien vous imprégner des deux «musiques » différentes. 
11 Maintenant vous n'allez en entendre qu'une, laquelle?

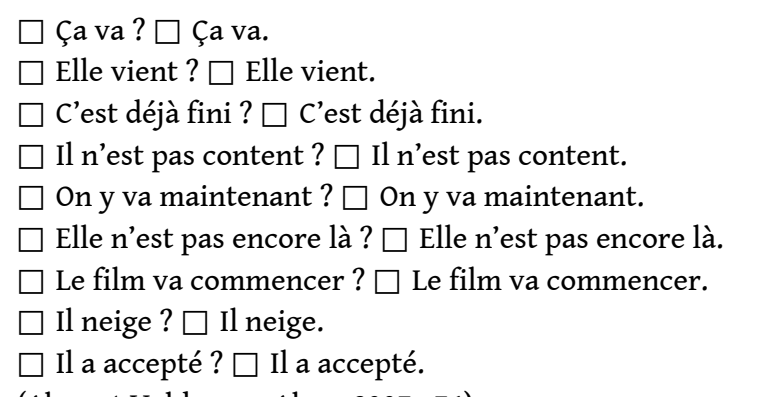

(Abry et Veldeman-Abry, 2007 : 74)

12 Puis, les apprenants répètent les deux types de phrases. Enfin le professeur travaille la production en interaction avec le groupe classe. Il prononce une des deux phrases du corpus avec une des deux intonations et les apprenants prononcent l'autre.

\section{$2^{\mathrm{e}}$ étape : Découverte du poème}

13 Observez ce poème. Marquez les groupes de souffle et la voyelle accentuée. Écoutez et vérifiez avec la lecture de l'acteur. Barrez toutes les lettres muettes du texte. Écoutez à nouveau.

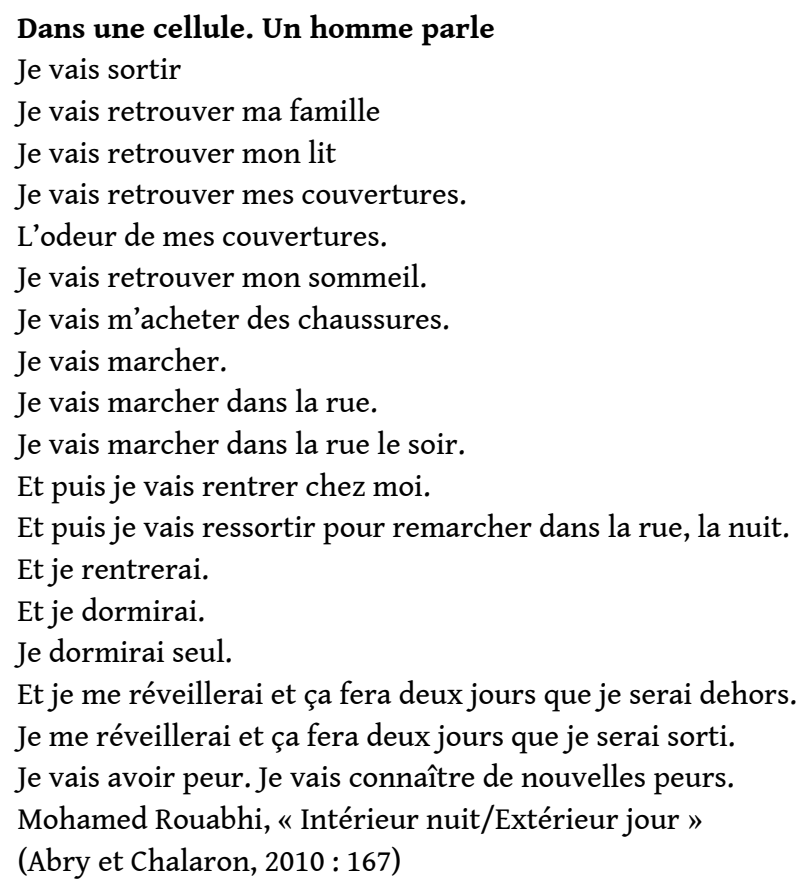

Le professeur travaille la compréhension du poème et fait ressentir la situation que vit cet homme qui va sortir de prison.

\section{$3^{\mathrm{e}}$ étape : Interprétation du poème}

Lisez-le seul en vous enregistrant. Dites-le doucement, en articulant lentement chaque syllabe. Puis écoutez votre production.

Une fois que le poème est bien maîtrisé par les apprenants, le professeur forme des groupes de 3 ou 4 apprenants et leur demande de lire le poème à plusieurs voix (changement à chaque vers ou à chaque groupe de souffle) ou à l'unisson. 
17 Variante : Ce poème comporte beaucoup de [R] dans toutes les positions. L'enseignant utilisera les différents mots pour faire acquérir sa prononciation. Il leur fait repérer tous les $[\mathrm{R}] \mathrm{du}$ texte pour les classer dans un tableau (position initiale, intervocalique, post-consonantique et finale).

Puis il leur fait répéter les [R] avec la progression suivante :

- en position finale, position moins tendue dans un souffle ;

- en fin de syllabe ;

- intervocalique, notamment tous les futurs ;

- le [R] post-consonantique.

19 L'enseignant fait remarquer l'allongement de toute voyelle en syllabe fermée devant $\mathrm{R}$ (ici, avec six timbres différents) : sortịr, couverture, chaussures, soir, jours, dehors, avoir, peur(s). Les mots seront répétés plusieurs fois. La durée des voyelles qui est de position en français est souvent oubliée. Il est nécessaire de travailler ce phénomène en le faisant entendre puis produire par les apprenants.

\section{Exemple 2}

\section{Objectifs :}

- s'approprier les différentes intonations. Développer la fluidité et la musicalité de la langue française avec la mise en voix d'un texte de Nina Bouraoui.

Niveau : A2.

\section{$1^{\text {re }}$ étape : Discrimination}

Deux exercices pour sensibiliser à la différence entre l'intonation de la question et de l'assertion (affirmative ou négative) pour le premier exercice et entre l'intonation de la question et de l'exclamation pour le second exercice.

1. Quel dialogue entendez-vous? Cochez.

\begin{tabular}{|c|c|c|c|c|}
\hline & $\begin{array}{l}\text { - Assertion } \\
\text { - Question? }\end{array}$ & & $\begin{array}{l}\text { - Question? } \\
\text { - Assertion }\end{array}$ & \\
\hline & $\begin{array}{l}\text { - C'est lui } \\
\text {-C'est lui? }\end{array}$ & $\mathrm{x}$ & $\begin{array}{l}\text { - C'est lui? } \\
\text {-C'est lui }\end{array}$ & \\
\hline & $\begin{array}{l}\text { - C'est possible. } \\
\text { - C'est possible? }\end{array}$ & & $\begin{array}{l}\text { - C'est possible? } \\
\text { - C'est possible. }\end{array}$ & X \\
\hline 1 & $\begin{array}{l}\text { Ça va aller. } \\
\text { Ça va aller? }\end{array}$ & & $\begin{array}{l}\text { Ça va aller? } \\
\text { Ça va aller }\end{array}$ & \\
\hline 2 & $\begin{array}{l}\text { Ce n'est pas fini. } \\
\text { Ce n'est pas fini? }\end{array}$ & & $\begin{array}{l}\text { Ce n'est pas fini? } \\
\text { Ce n'est pas fini. }\end{array}$ & \\
\hline 3 & $\begin{array}{l}\text { On aura le temps. } \\
\text { On aura le temps? }\end{array}$ & & $\begin{array}{l}\text { On aura le temps? } \\
\text { On aura le temps. }\end{array}$ & \\
\hline
\end{tabular}




\begin{tabular}{|l|l|l|l|}
\hline 4 & $\begin{array}{l}\text { Ça fait des histoires. } \\
\text { Ça fait des histoires? }\end{array}$ & $\begin{array}{l}\text { Ça fait des histoires ? } \\
\text { Ça fait des histoires. }\end{array}$ \\
\hline
\end{tabular}
question, un point d'exclamation pour un ordre.

[tytãva] Tu t'en vas?

[typarimedjatmã] Tu pars immédiatement !

1. [tyturnagof]

2. [tymesala]

3. [typoztakєstjõm $\tilde{\operatorname{tn}} \widetilde{]}]$

4. [vunpozepatkestjõ]

5. [vuvuzarete]

Corrigé : 1. Tu tournes à gauche? 2. Tu mets ça là !3. Tu poses ta question maintenant! 4. Vous ne posez pas de question? 5. Vous vous arrêtez !

(Abry et Chalaron, $2011: 14$ )

\section{$2^{\mathrm{e}}$ étape : Articulation (exercice d'écoute et de production orale au téléphone)}

Observez ces quatre extraits de dialogues. Quelle est la situation? Lisez et écoutez ces extraits. Répétez-les en même temps que le locuteur.

1. Allô... Oui, je suis arrivé... à deux heures... ça va très bien, oui... Je suis à l'aéroport... Mon train est à 16 heures... non, pas à 6 heures, à SEIZE heures... oui, 16 heures 06 exactement... SEI-ZEURES-SIX... J'arrive dans trois heures... Non, je prendrai un taxi... À tout à l'heure...

2. Allô ?... Lazare ?... Ça ne va pas ?... Qu'est-ce qu'il y a ? .... Elle est partie ? .... Elle est partie quand ?... Aujourd'hui ou hier ?... Tu es triste ?... Tu es où ?... Qu'est-ce que tu fais ?... Tu veux venir dîner ?... Tu as le code ?... 1789 oui ... À tout de suite. 3. C'est le Docteur Chamoud, oui, bonjour... Parlez, je vous écoute... Venez cet après midi... Alors venez tout de suite... Non, ne venez pas à pied... Appelez un taxi... Calmez-vous, calmez-vous... Non, n'appelez pas le SAMU... Donnez-lui une aspirine et attendez-moi... Non, n'appelez pas les pompiers... Calmez-vous... Je vais venir... J'arrive...

4. Allô... Encore !... Non, non, non et non, c'est NON... C'est CLAIR, non ?... Et, ne m'appelle plus... NE-M'A-PELLE-PLUS... tu comprends ?... Quoi ?!!!! Qu'est-ce que tu dis ?!!! ... Ah non ! Ca suffit !... Ne me dis plus JAMAIS ça... plus jamais...

(Abry et Chalaron, $2010: 22$ )

L'enseignant fait analyser plus précisément chaque situation et les différents types d'intonation: interrogative, déclarative, exclamative. Puis les apprenants choisissent l'un des dialogues et à deux, ils jouent le dialogue en entier. Comme ils n'entendent qu'un locuteur, il faut qu'ils imaginent les répliques de l'autre interlocuteur.

\section{$3^{\mathrm{e}}$ étape : Interprétation de l'extrait littéraire}

Écoutez le texte. Lisez-le ensuite avec l'intonation montante. À chaque espacement du texte, marquez un silence de deux secondes.

«Avez-vous des grains de beauté? Des cheveux blancs que vous teignez? Pratiquez-vous un sport? Prenez-vous des coups de soleil ? [...] Avez-vous peur de la nuit? De l'amour? Comment se prénomment vos enfants ? Êtes-vous une mère 
douce? Combien de baisers par jour? Quels sont vos mots sur moi ? Quel est mon dossier? Me trouvez-vous jolie? Intelligente? Perdue? Avez-vous fixé ma voix sur une bande magnétique ? Dois-je vous avouer qu'il m'arrive de rêver de vous?»

Nina Bouraoui, Mes mauvaises pensées (Abry et Veldeman-Abry, $2007: 76$ ) compréhension des questions, l'enseignant demande aux apprenants de lire le texte en respectant les espacements pour traduire "plus intensément que dans la ponctuation ordinaire les sentiments, les sensations ou les impressions de l'auteur» (Vielmas, 1990 : 29). S'il dispose d'un laboratoire de langue et d'une salle multimédia avec casques et micros, chaque apprenant doit s'enregistrer et se réécouter plusieurs fois sur certaines phrases ou sur la totalité de l'extrait. Ensuite, il se réécoute, compare sa production avec celle de l'acteur et s'auto-corrige, si nécessaire. Si l'enseignant n'a pas de laboratoire, il demande aux apprenants d'utiliser leur téléphone portable. Pour finir, les apprenants doivent mémoriser à la maison tout ou une partie du texte. Lors du cours suivant, ils récitent soit au laboratoire, soit en classe, ce qu'ils ont mémorisé sans le texte sous les yeux.

Puis nous continuerons avec quatre exemples de démarche permettant de travailler certains sons et spécificités du français.

\section{Exemple 3}

Objectif: s'approprier les trois voyelles nasales par la mise en voix d'un texte de Marguerite Duras.

Niveau : A2.

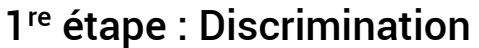

32 1. Dans quel ordre entendez-vous les mots suivants?

\begin{tabular}{|c|c|c|c|c|c|c|c|c|c|}
\hline & \multicolumn{4}{|c|}{ Syllabe ouverte } & & \multicolumn{4}{|c|}{ Syllabe fermée } \\
\hline & 1. $[\tilde{\varepsilon}]$ & 2. [ã] & 3. [õ] & & & 1. $[\tilde{\varepsilon}]$ & 2. [ã] & 3. [õ $]$ & \\
\hline Exemple & teint & temps & ton & 213 & Exemple & pinte & pente & ponte & 312 \\
\hline 1 & $\operatorname{lin}$ & lent & long & & & teinte & tente & tonte & \\
\hline 2 & sain & cent & son & & & mainte & menthe & monte & \\
\hline 3 & main & ment & mon & & & linge & lange & longe & \\
\hline 4 & rein & rang & rond & & & Reims & rance & ronce & \\
\hline 5 & bain & banc & bon & & & feinte & fente & fonte & \\
\hline
\end{tabular}




\section{$2^{\mathrm{e}}$ étape : Découverte et mise en voix du texte}

Lisez le texte. Repérez les voyelles nasales et classez-les dans le tableau.

L'homme élégant est descendu de la limousine, il fume une cigarette anglaise. Il regarde la jeune fille au feutre d'homme et aux chaussures d'or. Il vient vers elle lentement. C'est visible, il est intimidé. Il ne sourit pas tout d'abord. Tout d'abord il lui offre une cigarette. Sa main tremble. Il y a cette différence de race, il n'est pas blanc, il doit la surmonter, c'est pourquoi il tremble. Elle lui dit qu'elle ne fume pas, non merci. Elle ne dit rien d'autre, elle ne lui dit pas laissez-moi tranquille. Alors il a moins peur. Alors il lui dit qu'il croit rêver. Elle ne répond pas. Ce n'est pas la peine qu'elle réponde, que répondrait-elle. Elle attend. Alors il le lui demande : mais d'où venez-vous ? Elle dit qu'elle est la fille de l'institutrice de l'école de filles de Sadec.

Marguerite Duras, L'Amant

(Abry et Veldeman-Abry, $2007: 152$ )

L'enseignant dans un premier temps travaille la compréhension du texte. Puis, les apprenants en binômes repèrent les voyelles nasales dans le texte. L'enseignant corrige. Il fait ensuite répéter les mots trouvés en exagérant la position des lèvres, plus ou moins arrondies et projetées selon la voyelle prononcée : chaque apprenant répète un mot, deux ou trois avec une voyelle nasale différente (élégant/vient/non).

Pour finir les apprenants doivent lire cet extrait avec la voix douce et rassurante d'une hôtesse de l'air.

\section{Exemple 4}

Objectifs : Sensibiliser au registre familier par la mise en voix d'un texte d'Anna Gavalda. Comprendre les règles $\mathrm{du}$ « $\mathrm{e}$ » instable (chute et maintien).

Niveau : A2.

\section{$1^{\text {re }}$ étape : Discrimination}

Écoutez et cochez la phrase entendue. Puis entraînez-vous à prononcer ces phrases.

\begin{tabular}{|l|l|}
\hline Registre standard & Registre familier \\
\hline Je le prends & Je l' prends \\
\hline Il me demande & Il me d'mande \\
\hline Je me souviens & Je m' souviens \\
\hline Pas de reprise & Pas d' reprise \\
\hline Pas de demande & Pas d' demande \\
\hline
\end{tabular}

\section{$2^{\mathrm{e}}$ étape : Découverte du texte}

40 L'enseignant commence par un petit échange avec les apprenants en grand groupe. Il leur demande de se souvenir de leur enfance. 
41 Puis l'enseignant prépare le monologue du texte d'Anna Gavalda. Il met les apprenants en binômes et donne à chaque binôme une des 8 phrases du monologue avec «je me souviens...». Chaque binôme doit travailler la prononciation familière et la prononciation standard. Il passe entre les groupes pour les aider à prononcer.

Exemple :

Phrase 1: «Je me souviens que t'avais dix ans, que tu mesurais 1 mètre 29, que tu pesais 26 kilos, que t'avais eu les oreillons l'année d'avant, je m'en souviens de la visite médicale »

$\rightarrow J^{\prime}$ me souviens qu't'avais dix ans, qu' tu mesurais 1 mètre 29 , qu' tu pesais 26 kilos, qu' t'avais eu les oreillons l'année d'avant.

\section{$3^{e}$ étape : Mise en voix du texte}

Lisez le texte. Jouez le dialogue à deux, une fois en registre standard et une fois en registre familier.

«-C'est vrai que tu ne te souviens pas de moi?

- Non, c'est pas vrai.

- Tu t'en souviens?

- Oui.

- Tu te souviens de quoi?

- Je me souviens que t'avais dix ans, que tu mesurais 1 mètre 29 , que tu pesais 26 kilos, que t'avais eu les oreillons l'année d'avant, je m'en souviens de la visite médicale. Je me souviens que t'habitais à Choisy-le-Roi et à l'époque, ça m'aurait coûté 42 francs de venir te voir en train. Je me souviens que ta mère s'appelait Catherine et ton père Jacques. Je me souviens que t'avais une tortue d'eau qui s'appelait Candy et ta meilleure copine avait un cochon d'Inde qui s'appelait Anthony. Je me souviens que tu avais un maillot de bain vert avec des étoiles blanches et ta mère t'avait même fait un peignoir avec ton nom brodé dessus. Je me souviens que tu avais pleuré un matin parce qu'il n'y avait pas de lettres pour toi. Je me souviens que tu t'étais collé des paillettes sur les joues le soir de la boum et qu'avec Rebecca, vous aviez fait un spectacle sur la musique de Grease...

- Oh, là, là, mais c'est pas croyable la mémoire que tu as !»

Anna Gavalda, Je voudrais que quelqu'un m'attende quelque part, 1999.

(Abry et Chalaron, $2011: 130$ )

Enfin nous voudrions terminer par le slam et, avec la notion de style oral telle que la définit Claude Hagège (1985:84) : «Le style oral est un véritable genre littéraire. Il s'agit d'une tradition culturelle qui parait apporter une justification à la création d'un terme, orature, lequel deviendrait symétrique de celui d'écriture entendue comme littérature ».

\section{Exemple 5}

Objectifs : s'approprier le phénomène de la diérèse avec l'extrait d'un slam de Grand Corps Malade (Vorger, Abry et Bouchoueva, $2016: 58$ et 97).

Niveau : A2.

La diérèse, c'est la prononciation en deux syllabes d'une séquence de deux sons prononcés habituellement en une syllabe. Mais c'est la règle lorsque le « $\mathrm{i}$ » est précédé de deux consonnes. Exemple: "oublier»[ublije] se prononce en trois syllabes et « confier » en 2 syllabes [kõfje]. Pour les autres voyelles, on peut la faire ou non : nuage [nya3] ou [nuaz] (une ou deux syllabes). 
47 L'enseignant commence le cours en demandant aux apprenants ce qu'ils ont oublié ce matin ou ce qu'ils ont l'habitude d'oublier.

\section{$1^{\text {re }}$ étape : Discrimination}

\begin{tabular}{|l|l|l|}
\hline & {$[\mathrm{i}]$} & {$[\mathrm{ij}]$} \\
\hline oublier & & \\
\hline étudier & & \\
\hline apprécier & & \\
\hline crier & & \\
\hline skier & & \\
\hline photocopier & & \\
\hline supplier & & \\
\hline
\end{tabular}

Puis écoutez et répétez les phrases suivantes.

Nous vous prions de bien vouloir nous excuser de ce retard.

Je voudrais me marier cet automne.

Elle pense publier son livre l'année prochaine.

Vous allez recopier votre lettre.

\section{$2^{\mathrm{e}}$ étape : Découverte et mise en voix du slam}

50 L'enseignant présente le slam et le slameur Grand Corps Malade. Il fait écouter le début du slam (<https://www.dailymotion.com/video/x6ugwl>) avec le texte sous les yeux. Il travaille la compréhension du texte puis leur demande de le mémoriser.

51 Par groupe de 2 ou 3, interprétez-le à plusieurs voix.

J'ai oublié de commencer ce texte par une belle introduction

J'ai oublié de vous préparer avant d'entrer en action

J'ai oublié de vous prévenir que je m'aperçois que dans ma vie

J'ai oublié pas mal de choses si vous voulez mon avis

J'ai oublié d'être sage, j'ai oublié d'être prudent

J'ai oublié de me ménager et je me suis cassé les dents.

Grand Corps Malade, «J'ai oublié

\section{Exemple 6}

Objectifs : s'approprier les phénomènes des enchaînements et des liaisons avec l'extrait du slam d'Abd Al Malik. 


\section{$1^{\text {re }}$ étape : Discrimination et entraînement}

Lisez et écoutez l'exemple suivant :

[z] enfants: les enfants, des enfants, mes enfants, tes enfants, ces enfants, ses enfants, nos enfants, vos enfants, leurs enfants, aux enfants, plusieurs enfants, quelques enfants, certains enfants, de nombreux enfants

[n] enfant : mon enfant, ton enfant, son enfant, un enfant, aucun enfant

$[\mathrm{t}]$ : tout enfant.

À votre tour, entraînez-vous avec les mots proposés comme dans l'exemple : ami, objet, examen, intérêt. (Abry et Veldeman-Abry, 2007 : 92)

\section{$2^{\mathrm{e}}$ étape : Découverte et mise en voix du slam} émotionnelle qui peuvent séduire les apprenants de tous niveaux et de tous âges. À l'enseignant de savoir choisir le type de texte et d'apprécier son niveau de difficultés. Une langue, c'est d'abord un matériau sonore. Tout lecteur « entend » en même temps qu'il lit un texte. Il est important que l'apprenant étranger acquière une bonne prononciation des sons, une bonne syllabation avec les phénomènes d'enchaînement et de liaison, un bon rythme, une bonne intonation linguistique ou expressive. Comme dans la pièce célèbre de Bernard Shaw, Pygmalion, la qualité de la prononciation d'Eliza Doolittle est obtenue grâce à la mise en bouche répétée et la mémorisation d'un certain nombre de phrases et de textes mais surtout grâce à sa motivation, sa sensibilité et sa créativité. Le choix judicieux des textes littéraires en fonction des phénomènes phonétiques et de leur sensibilité constitue une tâche délicate pour l'enseignant. Mais, l'apprenant sera ravi de les découvrir et de se délier la langue avec ces jeux de langage, ces assonances et ces allitérations que les écrivains et notamment les poètes aiment introduire dans leurs écrits. 


\section{BIBLIOGRAPHIE}

ABRY D. et VELDEMAN-ABRY J., 2007, La phonétique : audition, prononciation, correction, Paris, CLE International, coll. « Techniques et pratiques de classe ».

ABRY D. et CHALARON M. L., 2010, Les 500 exercices de phonétique : niveau A1-A2, Paris, Hachette FLE.

-, 2011, Les 500 exercices de phonétique : niveau B1-B2, Paris, Hachette FLE.

HAGÈGE C., 1985, L'homme de paroles. Contribution linguistique aux sciences humaines, Paris, Fayard, coll. « Le temps des sciences».

VIELMAS M., 1990, À haute voix, Paris, CLE International, coll. « Techniques de classe ».

VORGER C., ABRY D. et BOUCHOUEVA K., 2016, Jeux de slam. Ateliers de poésie orale, Grenoble, PUG, coll. « Les outils malins du FLE ».

YAGUELLO M. (dir.), 2003, Le Grand livre de la langue française, Paris, Éditions du Seuil.

Æuvres citées

ABD AL MALIK, 2006, « Adam et Ève », album Gibraltar.

BOURAOUI N., 2005, Mes mauvaises pensées, Vanves, Stock, coll. « La Bleue ».

DURAS M., 1984, L’Amant, Paris, Les Éditions de Minuit.

FLAUBERT G., CEuvres complètes, Saint Julien en Genevois, Arvensa Éditions, pack numérique.

GAVALDA A., 1999, Je voudrais que quelqu'un m'attende quelque part, Paris, Le Dilettante.

GRAND CORPS MALADE, 2006, « J'ai oublié », album Midi 20.

GREEN J., 1985, Le langage et son double, Paris, Fayard.

MIZUBAYASHI A., 2011, Une langue venue d'ailleurs, Paris, Gallimard, coll. « Folio ».

PENNAC D., 1992, Comme un roman, Paris, Gallimard, coll. « Blanche ».

ROUABHI M., 2000, « Intérieur nuit /Extérieur jour », dans Des mots pour la vie. Pièces courtes, Paris, Pocket.

\section{RÉSUMÉS}

Depuis l'Approche Communicative et aujourd'hui avec la méthode actionnelle, la littérature a été réhabilitée. Elle fait partie des supports de cours mais reste néanmoins réservée aux niveaux plus avancés du Cadre européen commun de référence pour les langues (CECRL). Nous montrerons à travers plusieurs exemples qu'au contraire l'apprenant de FLE doit être sensibilisé dès le niveau débutant à la musicalité de la langue française par des textes où les auteurs se sont attachés à en travailler tout particulièrement le rythme, l'intonation, la syllabation et la sonorité. Nous montrerons aussi qu'il faut aller au-delà de la phonématique pure et de la discrimination auditive par paires minimales et traiter de phénomènes que l'on a eu trop tendance à oublier mais qui ont reçu l'attention de certaines nouvelles phonologies qui se sont développées pour le domaine français : le « e » instable, la quantité vocalique, les enchaînements, la diérèse... 
Since the time of communicative approach to today's action-oriented approach, the literature has been reclaimed. It remains a part of the course material however is reserved for more advanced levels of CEFR. We, on the contrary, illustrate with several examples that the learner of French as foreign language (FLE) should be acquainted to the musicality of French language from the beginner level itself, with the help of texts in which the authors have worked on rhythm, intonation, syllabation and sounds. We also demonstrate that it is necessary to go beyond pure phonematics and auditory discrimination in minimal pairs and to deal with phenomena that has been omitted but nonetheless has received the responsiveness of certain new phonologies that are developed for French domain: the unstable « $\mathrm{e}$ », the vocalic quantity, the sequences, the dieresis...

\section{INDEX}

Keywords : Phonetics and phonology, literature, the CEFR levels, non-functional phenomena, phonetic correction

Mots-clés : phonétique et phonologie, littérature, niveaux du CECRL, phénomènes non fonctionnels, correction phonétique

\section{AUTEURS}

\section{JULIE VELDEMAN-ABRY}

Université catholique de Lyon

DOMINIQUE ABRY

Université Grenoble Alpes 